\title{
Spontaneous inelastic Rayleigh scattering in optical fibers
}

\author{
Olukayode Okusaga, ${ }^{1, *}$ James P. Cahill,,${ }^{1,2}$ Andrew Docherty, ${ }^{2}$ Curtis R. Menyuk, ${ }^{2}$ and Weimin Zhou ${ }^{1}$ \\ ${ }^{1}$ Sensors and Electronic Devices Directorate, U.S. Army Research Laboratory, 2800 Powder Mill Road, Adelphi, Maryland 20783, USA \\ ${ }^{2}$ Computer Science and Electrical Engineering Department, University of Maryland Baltimore Country, 1000 Hilltop Circle, \\ Baltimore, Maryland 21250, USA \\ *Corresponding author: olukayode.k.okusaga.civ@mail.mil
}

Received September 14, 2012; revised January 14, 2013; accepted January 16, 2013; posted January 17, 2013 (Doc. ID 176303); published February 13, 2013

Rayleigh scattering (RS) adds noise to signals that are transmitted over optical fibers and other optical waveguides. This noise can be the dominant noise source in a range between $10 \mathrm{~Hz}$ and $100 \mathrm{kHz}$ from the carrier and can seriously degrade the performance of optical systems that require low close-in noise. Using heterodyne techniques, we demonstrate that the backscattered close-in noise spectrum in optical fibers is symmetric about the carrier and grows linearly with both input power and fiber length. These results indicate that the RS is spontaneous and is due to finite-lifetime thermal fluctuations in the glass. (C) 2013 Optical Society of America

OCIS codes: $190.4370,290.5870$.

A new class of radio-frequency (RF)-photonic applications uses lasers and optical media, such as optical fibers to generate, transmit, and process ultralow-noise RF signals. The performance of systems, such as optoelectronic oscillators, optical-fiber time and frequency-transfer systems, microresonator-based oscillators, and fiber sensors is limited in part by scattering in the optical media [1-4]. It has been shown that Rayleigh scattering (RS) induces noise in a $10-100 \mathrm{kHz}$ bandwidth around the optical carrier in optical fibers [트, $\underline{6}]$.

Previously, we measured the Rayleigh backscattered noise spectrum in single-mode optical fibers, and we showed that the gain relative to the input relative intensity noise (RIN) matched the theoretically expected spectrum for stimulated RS in bulk materials, but with a much narrower spectrum [7]. A narrower spectrum is expected from processes that are driven by the transverse intensity gradient of the light in the optical fiber, as is the case for guided acoustic wave Brillouin scattering (GAWBS). For this reason, we refer to the Rayleigh scattering process in this case as guided entropy mode Rayleigh scattering (GEMRS). Our work was carried out using standard SMF-28e communication fiber.

The experiments to date have not resolved the important question of whether we are observing a stimulated process or a spontaneous process. While the gain spectrum that we observed is reminiscent of a stimulated process, the analogous process of GAWBS is a spontaneous process. The answer to this question has profound implications for the physical origin of the scattering and the best methods for suppressing it. If the process is stimulated, then the source of the noise is amplification of the laser noise. In this case, the output noise spectrum should be asymmetric about the carrier. If it is upshifted, then we would infer that the scattering is primarily electroabsorptive; if it is downshifted, then we would infer that the scattering is primarily electrostrictive []․ In either case, we anticipate an exponential growth of the output spectrum as the input light power or the length of the fiber increases. By contrast, if the process is spontaneous, then the carrier is scattered by finite-lifetime thermal fluctuations in the optical fiber-as opposed to the frozen-in thermal fluctuations that have an infinite lifetime. A finite lifetime is required to account for the nonzero bandwidth and hence the slight inelasticity of the scattering. In this case, the output phase noise spectrum should be symmetric about the carrier, and the output spectrum should grow linearly as the input light power grows and the fiber length increases.

In this Letter, we demonstrate that the output noise spectrum is symmetric about the carrier and that the output power spectral density is linearly proportional to both the input laser power and the fiber length. This work strongly supports the hypothesis that we are observing a spontaneous process and is an important step toward obtaining a complete physical description of GEMRS.

Our work was carried out using standard SMF-28e communication fiber. In earlier work, Zhu et al. have found evidence of stimulated RS in specialty Brillouinsuppressed fiber $[\underline{9}, \underline{10}]$. Such fibers may prove useful in applications such as laser mode selectors [9].

The key difficulty with our prior homodyne experiments [7] was that our experimental technique was one-sided and thus could not distinguish between the upshifted and the downshifted spectrum. In this Letter, we use a heterodyne fiber-optic interferometer to measure both the sign and the magnitude of the backscattered noise spectrum. Figure 1 shows a schematic diagram of our heterodyne interferometer. The output signal from a semiconductor laser is split in two using a $75 \% / 25 \%$ optical splitter. Both output

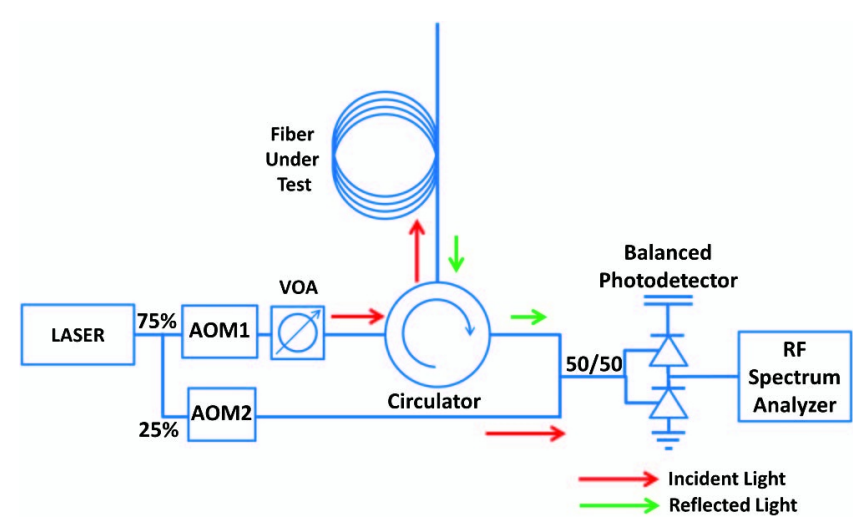

Fig. 1. Schematic diagram of our heterodyne interferometer. The acousto-optic modulators AOM1 and AOM2 are tuned to determine the IF. 
signals from the splitter are sent into acousto-optic modulators (AOMs) that can shift the frequency of the light by $75 \mathrm{MHz} \pm 5 \mathrm{MHz}$. The difference between the frequency shifts applied by each AOM is the intermediate frequency (IF) at which we measure the scattered spectrum. After the AOMs, the portion of the laser beam that passes through the $75 \%$ output port is sent through a variable optical attenuator (VOA), then through a circulator, and into the fiber under test. This beam serves as the pump for our Rayleigh measurements. The backscattered signal travels through the circulator and into one port of an optical 2-by-2 combiner. The portion of the laser beam that passes through the $25 \%$ output port is sent into the other input port of the 2-by-2 combiner. This signal serves as the local oscillator (LO) in our interferometer. The output signals from the combiner are sent to a balanced photodetector. The output current from the photodetector is proportional to the product of the backscattered signal and the LO. The photodetector signal is then sent to a fast Fourier transform (FFT) analyzer where we measure the spectrum. We chose the frequency shifts at the AOMs so that the IF lies within the $10 \mathrm{MHz}$ bandwidth of the FFT analyzer. The precise value of the IF did not alter our results.

We divide the measured backscattered spectrum by the intensity noise spectrum of the incident laser beam to determine the Rayleigh gain [7]. Figure 2 shows a plot of the Rayleigh backscattered spectrum from a $10 \mathrm{~km}$ length of single-mode fiber with a $0 \mathrm{dBm}$ incident beam measured with a $1.5 \mathrm{MHz}$ IF. The abscissa on this plot is the offset frequency from the IF in kilohertz. We refer to the negative offset frequency region as the "Stokes" region and to the positive offset frequency region as the "anti-Stokes" region. The noise spectrum is symmetric around the IF. This symmetry is analogous to spontaneous Brillouin scattering where the Stokes and anti-Stokes peaks are equal in intensity. This data suggests that the Rayleigh backscattered spectrum does not experience coherent exponential growth with distance in fibers. In Fig. 2 and all subsequent plots we used a logarithmic scale for the abscissa so as to show the spectrum from $10 \mathrm{~Hz}$ to $1 \mathrm{MHz}$. In addition, we folded the Stokes and anti-Stokes curves on top of each other so that the abscissa represents the absolute value of the offset frequency from the IF.

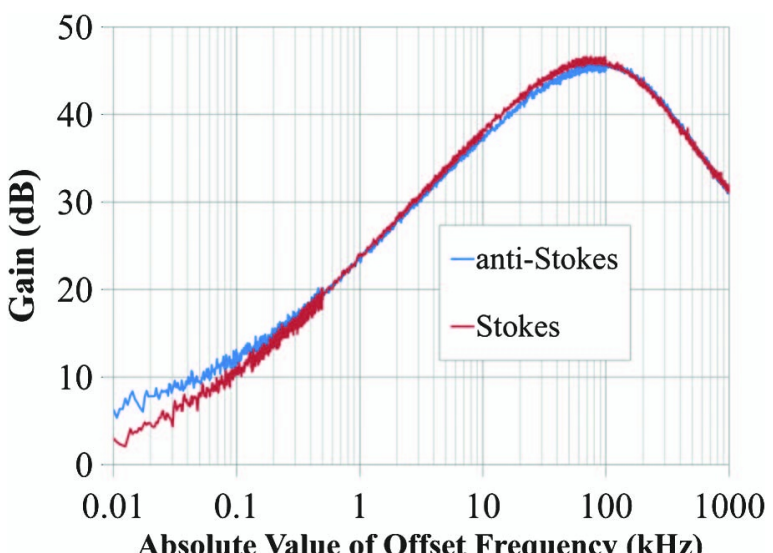

Fig. 2. Plots of the measured Rayleigh gain from a $10 \mathrm{~km}$ single-mode fiber with a $0 \mathrm{dBm}$ incident laser beam.
Doing so allows us to more clearly determine the symmetry of the gain spectra.

We investigated whether the symmetry of the Rayleigh gain spectrum changes with incident power. To do so, we varied the input power into a $10 \mathrm{~km}$ length of singlemode fiber using a VOA. Figure 3 shows plots of the Rayleigh gain spectrum for incident power levels from 0 to $12 \mathrm{dBm}$. These plots are normalized to the incident power so that a linear increase in scattered power appears as constant gain. The data show that RS is linear in optical power over the frequency range from $10 \mathrm{~Hz}$ to $1 \mathrm{MHz}$ around the frequency of the incident light. These results are what are expected with a spontaneous scattering process.

We also measured the Rayleigh gain as the fiber length changed. We did so by injecting an $8 \mathrm{dBm}$ laser beam into various lengths of single-mode fiber. Figure 4 shows plots of the Stokes and anti-Stokes gain spectra for $40 \mathrm{~m}, 1 \mathrm{~km}$, and $10 \mathrm{~km}$ fibers. The spike at $24 \mathrm{kHz}$ offset frequency is due to electrical noise in our measurement system and is present in the $40 \mathrm{~m}$ and $1 \mathrm{~km}$ Stokes and antiStokes curves. The electrical noise spike is not visible in the $10 \mathrm{~km}$ Stokes and anti-Stokes curves because the absolute value of the RS-induced noise at that offset frequency is greater than the magnitude of the system noise spike. Figure $\underline{5}$ shows plots of the peak Stokes and anti-Stoke gain versus fiber length. Our measurement

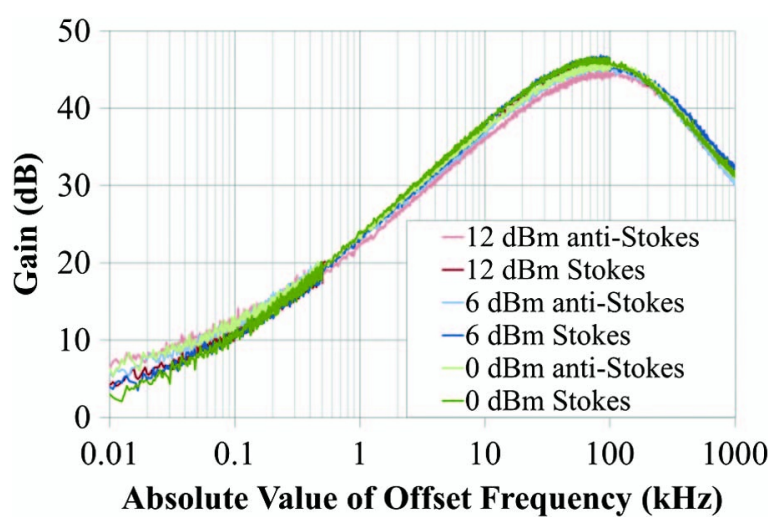

Fig. 3. Plots of the measured Rayleigh gain from a $10 \mathrm{~km}$ single-mode fiber for various incident power levels. The gain curves were normalized to the incident optical power.

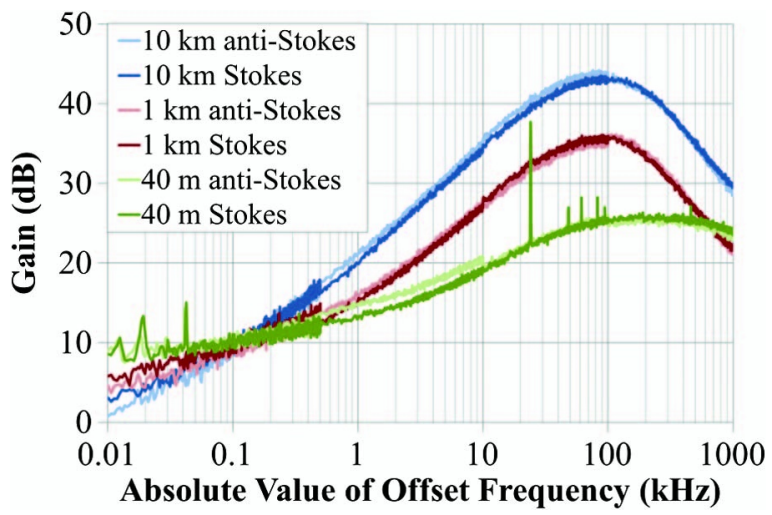

Fig. 4. Plots of the measured Rayleigh gain from $40 \mathrm{~m}, 1 \mathrm{~km}$, and $10 \mathrm{~km}$ single-mode fibers with an $8 \mathrm{dBm}$ incident laser beam. 


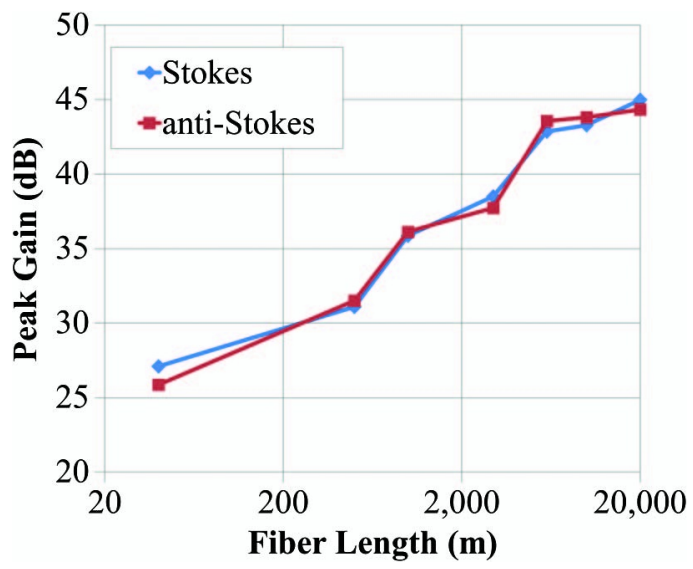

Fig. 5. Plots of the Stokes and anti-Stokes gain peaks versus fiber length for an $8 \mathrm{dBm}$ incident laser beam.

system was incapable of detecting the RS spectrum from lengths of single-mode fiber much shorter than $40 \mathrm{~m}$ due to the RIN of our pump laser. Our data shows that the Rayleigh gain scales linearly with length. Again, this result is consistent with a spontaneous process.

In this Letter, we have carried out heterodyne measurements to determine whether backscattered GEMRS in single-mode optical fibers is spontaneous or stimulated. The output spectrum of Corning SMF-28e fiber is symmetric about the carrier and grows linearly with both input laser power and fiber length. These results indicate that the process is spontaneous. These results should point the way to a complete physical understanding of GEMRS. More importantly, these results have important implications for low-noise RF-photonic applications, such as optoelectronic oscillators, time-frequency transfer over optical fibers, microresonators, and precision Doppler radar remoting. An understanding of GEMRS suggests ways of controlling or suppressing it that will be the subject of future studies.

The authors thank Dr. James Hanssen and Dr. Christopher Ekstrom of the U.S. Naval Observatory for their invaluable assistance in developing the heterodyne optical interferometer. Work at UMBC was supported by DARPA, ARL, and MPCA.

\section{References}

1. O. Okusaga, W. Zhou, E. Levy, M. Horowitz, G. M. Carter, and C. R. Menyuk, in Proceedings of the Conference on Lasers and Electro-Optics (Optical Society of America, 2009), paper CFB3.

2. P. A. Williams, W. C. Swann, and N. R. Newbury, J. Opt. Soc. Am. B 25, 1284 (2008).

3. A. B. Matsko, Practical Applications of Microresonators in Optics and Photonics (CRC, 2009).

4. M. Froggat and J. Moore, Appl. Opt. 37, 1735 (1998).

5. W. K. Burns and R. P. Moeller, J. Lightwave Technol. 1, 381 (1983)

6. T. Zhu, X. Bao, L. Chen, H. Liang, and Y. Dong, Opt. Express 18, 22958 (2010).

7. O. Okusaga, J. Cahill, A. Docherty, W. Zhou, and C. R. Menyuk, Opt. Lett. 37, 683 (2012).

8. R. W. Boyd, Nonlinear Optics, 3rd ed. (Elsevier, 2008), Chap. 9.6. see, in particular, Fig. 9.6.1.

9. T. Zhu, X. Bao, and L. Chen, J. Lightwave Technol. 29, 1802 (2011).

10. T. Zhu, X. Bao, and L. Chen, Opt. Commun. 285, 1371 (2012). 